ARTICLE

\title{
Redundant and non-redundant cytokine-activated enhancers control Csn1s2b expression in the lactating mouse mammary gland
}

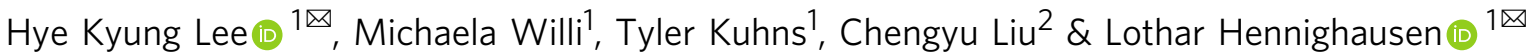

Enhancers are transcription factor platforms that synergize with promoters to control gene expression. Here, we investigate enhancers that activate gene expression several hundredfold exclusively in the lactating mouse mammary gland. Using ChIP-seq for activating histone marks and transcription factors, we identify two candidate enhancers and one superenhancer in the Csn1s2b locus. Through experimental mouse genetics, we dissect the lactation-specific distal enhancer bound by the mammary-enriched transcription factors STAT5 and NFIB and the glucocorticoid receptor. While deletions of canonical binding motifs for NFIB and STAT5, individually or combined, have a limited biological impact, a non-canonical STAT5 site is essential for enhancer activity during lactation. In contrast, the intronic enhancer contributes to gene expression only in late pregnancy and early lactation, possibly by interacting with the distal enhancer. A downstream super-enhancer, which physically interacts with the distal enhancer, is required for the functional establishment of the Csn1s $2 b$ promoter and gene activation. Lastly, NFIB binding in the promoter region fine-tunes Csn1s2b expression. Our study provides comprehensive insight into the anatomy and biology of regulatory elements that employ the JAK/STAT signaling pathway and preferentially activate gene expression during lactation.

\footnotetext{
${ }^{1}$ Laboratory of Genetics and Physiology, National Institute of Diabetes and Digestive and Kidney Diseases, US National Institutes of Health, Bethesda, MD 20892, USA. ${ }^{2}$ Transgenic Core, National Heart, Lung, and Blood Institute, US National Institutes of Health, Bethesda, MD 20892 , USA.

凶email: hyekyung.lee@nih.gov; lotharh@niddk.nih.gov
} 
E nhancers are transcription component platforms that control the location, timing and intensity of gene expression ${ }^{1,2}$. While current approaches, such as the ChIP-seq and physical contact studies, are useful in identifying candidate enhancers, their biological predictions are limited and validation through genetic experiments is needed. Enhancers are occupied by multiple transcription factors (TFs) that might bind directly to DNA through their respective recognition motifs or indirectly through tethering. Since experimental genetic studies generally ablate the entire enhancer, the structural and functional contribution of individual TFs remains to be understood.

Several hundred genes are uniquely expressed in mammary tissue and activated by pregnancy and lactation hormones through the tyrosine kinase JAK2 and the transcription factors Signal Transducer and Activator of Transcription (STAT) 5A and $5 \mathrm{~B}$ (referred to as STAT5) ${ }^{3-5}$. STAT5 is activated by prolactin and it controls mammary alveolar development during pregnancy and the activation of genetic programs resulting in lactation $^{3,4}$. While most STAT5 target genes are highly induced during pregnancy and to a lesser extent during lactation ${ }^{6}$, the activation of the Csn1s $2 b$ gene $^{7}$ occurs preferentially during lactation $^{8}$, possibly through enhancers that are specifically established after parturition ${ }^{8}$. ChIP-seq profiles for STAT5 and $\mathrm{H} 3 \mathrm{~K} 27 \mathrm{ac}$ and other mammary-enriched TFs suggested the presence of highly complex mammary enhancers ${ }^{8}$. Although most of these enhancers appear to depend on STAT5 as the anchor for the establishment of larger protein complexes, the stage-specific generation of enhancers remains to be understood. It is not known why seemingly structurally identical enhancers can be activated by pregnancy hormones either during pregnancy or lactation.

Caseins, the major components of milk, are cardinal proteins that are unique to mammals. They are evolved from secretory calcium-binding phosphoproteins (SCPP) with the odontogenic ameloblast-associated (ODAM) gene being possibly a founding member. While a CSN3-like protein is already found in early amniotes and appears to be the first member of the family of five caseins, CSN1s2b is a more recent addition that evolved through gene duplication ${ }^{9}$. The mouse casein locus spans $\sim 400 \mathrm{kbp}$ and consists of five casein genes (Csn1s1, Csn2, Csn1s2a, Csn1s2b and Csn3) and at least three SCPP genes (Prr27, Odam and Fdcsp) that are expressed in salivary glands and possibly other secretory tissues. The casein locus remains a fertile ground for exploring tissue-restricted and hormone-controlled gene regulation. Foremost, while the casein genes are expressed exclusively in mammary tissue and are induced by pregnancy and lactation hormones, the interspersed genes are expressed preferentially in salivary gland tissue. Among the five casein genes, expression of $C s n 1 s 2 b$ is uniquely different from the other four in that its activation predominantly occurs during lactation and not during pregnancy. The evolution of the five caseins through gene duplication begs the question to what extent regulatory elements were duplicated, developed de novo or even shared between genes. It seems plausible that regulatory elements controlling the ancient SCCP genes were repurposed and acquired features that permitted their activity in secreting mammary gland cells. It also remains to be determined whether regulatory elements controlling the ancestral $C s n 3$ gene were acquired by the younger $C s n 1 s 2 b$ gene, which is separated from Csn 3 by three SCCP genes.

Here, we used ChIP-seq for activating histone marks and transcription factors to identify candidate enhancers in mammary tissue during pregnancy and lactation. We identified two candidate enhancers and one super-enhancer in the extended Csn $1 s 2 b$ locus and investigated a potential synergy between the prolactininduced TF STAT5 and the mammary-enriched Nuclear Factor I $\mathrm{B}$ (NFIB) in the establishment of lactation-specific regulatory elements. For this, we employed experimental mouse genetics and functionally dissected the two enhancers, the super-enhancer and the $\operatorname{Cs} n 1 s 2 b$ promoter. This permitted us to define the contributions of individual enhancers and the significance of STAT5 and NFIB in the activating the Csn1s $2 b$ gene during pregnancy and lactation.

\section{Results}

A Csn 1s $2 b$ distal enhancer is activated in mammary tissue during lactation. The five casein genes, positioned within a $\sim 400$ kbp locus, are expressed exclusively in mammary tissue under the control of pregnancy and lactation hormones (Supplementary Table 1). Interspersed in this locus are three genes that are preferentially expressed in salivary glands. While four out of the five casein genes are highly induced during pregnancy, $C s n 1 s 2 b$ is activated preferentially, and up to several-hundred-fold, during lactation, suggesting the presence of distinct regulatory elements. The adjacent $C s n 1 s 2 a$ and $C s n 1 s 2 b$ genes, which arose by geneduplication prior to the split of eutherian mammals ${ }^{10,11}$, are subject to a different regulation. While $C s n 1 s 2 b$ expression increased more than 250-fold between day 1 of lactation (L1) and day 10 (L10), Csn1s $2 a$ expression increased approximately 6-fold (Fig. 1a) suggesting the presence of regulatory elements that uniquely respond to lactation stimuli, with prolactin the most prominent hormone. To identify such putative regulatory elements, we dug deeper and used ChIP-seq profiling for transcription factor binding and the presence of activating histone marks (Fig. 1b-d and Supplementary Fig. 1a-c). Binding of STAT5A/B (referred to as STAT5), transcription factors activated by prolactin, was detected at three sites upstream of the Csn $1 s 2 a$ gene and at two sites at the Csn1s2b gene (Fig. 1b). Each of the five sites bound by STAT5 coincided with at least one GAS motif (the sequence recognized by STAT family members) supporting a direct protein-DNA interaction. The most proximal STAT5 binding sites at the two genes are close to the TSS, suggesting that they could be part of a combined promoter-enhancer unit. While maximum STAT5 binding at the Csn1s2a sites was already observed at day 18 of pregnancy (p18) and remained high throughout lactation, STAT5 binding at the candidate Csn $1 s 2 b$ enhancer was marginally detectable at p18 and was fully established between L1 and L10 (Fig. 1b and Supplementary Fig. 1a-c). Pol II loading and H3K4me3 coverage at the two loci also reflects the differential expression of the two genes (Fig. 1b-d).

A candidate distal enhancer (DE) bound by STAT5 and other TFs, including the glucocorticoid receptor (GR), NFIB and MED1, was identified $2.3 \mathrm{~kb} 5^{\prime}$ of the Csn1s $2 b$ TSS (Fig. 1d). STAT5 and NFIB binding coincided with their respective recognition motifs (TTCnnnGAA for STAT5 and TGGCA/ TGCCA for NFIB), suggesting direct protein-DNA interactions. One GR half site motif (TGTYCY/RGRACA) ${ }^{12-14}$ was identified within the DE and overlapped with the GAS motif in STAT5 binding site S1 (Supplementary Table 3). Putative GR motifs were located within the promoter binding site and also in two out of the three sites at the neighboring Csn1s2a gene (Fig. 1c). While unbiased motif searches for the mammary-enriched TFs STAT5 and NFIB have been conducted in mammary tissue from lactating mouse, no such information was available for the GR. We therefore performed a de novo motif search using GR ChIP-seq data from L10 mammary tissue (Supplementary Fig. 2). Out of the approximately 26,000 sites bound by the GR, 22,675 coincided with $\mathrm{H} 3 \mathrm{~K} 27 \mathrm{ac}$ marks, indicative of candidate regulatory elements. Motifs for transcription factors (ETS factors, STAT5 and Nuclear Factor I family) known to control mammary development and function were significantly enriched at the 22,617 sites bound by GR and marked by H3K27ac ( $\pm 500 \mathrm{bp}$ ). 

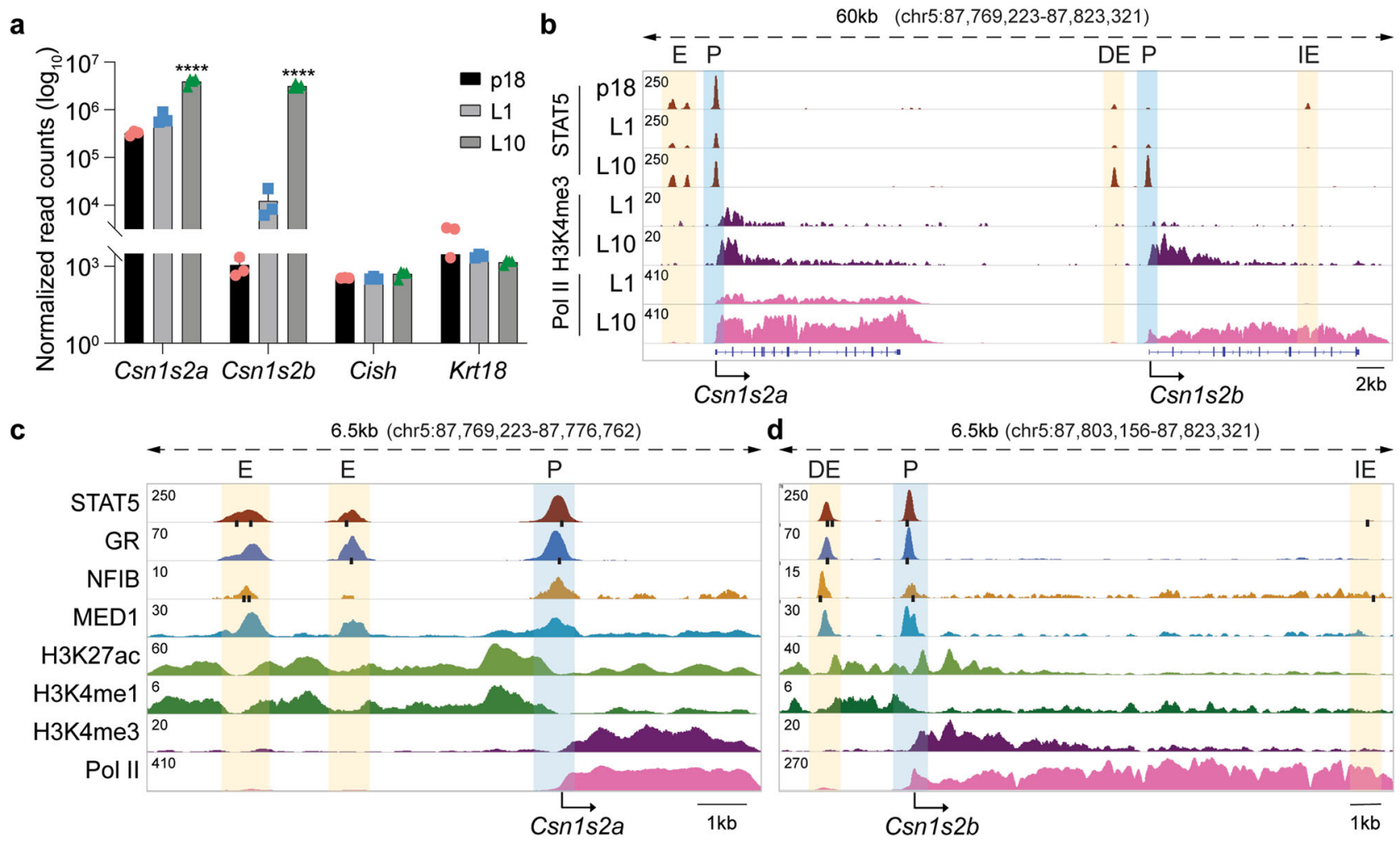

Fig. 1 Chromatin structures and activity of the Csn1s2a/b locus. a mRNA levels were measured by RNA-seq at day 18 of pregnancy (p18), day one of lactation (L1) and L10. Results are shown as the means \pm s.e.m. of independent biological replicates (p18 and L1, $n=3 ; \mathrm{L} 10, n=4$ ). Two-way ANOVA followed by Tukey's multiple comparisons test was used to evaluate the statistical significance of differences. The Cish and Krt18 genes served as controls. b ChIP-seq data for STAT5a binding, H3K4me3 marks and Pol II loading at the Csn1s2a-Csn1s2b locus during pregnancy (p18) and lactation (L1 and L10). The orange shades indicate candidate enhancers and the blue shades are promoter regions. The Cish locus served as control (Supplementary Fig. 1). DE, Csn1s2b distal enhancer; IE, intronic enhancer; P, promoter. c-d TF binding and activating histone marks at the Csn1s2a (c) and Csn1s2b (d) genes at L10. The black bars indicate DNA binding motifs for STAT5 (TTCnnnGAA), GR (RGXACAnnnTGTXCY) and NFIB (TGGCA/TGCCA). The orange and blue shades indicate putative enhancers and promoters, respectively.

GR ChIP-seq peaks were enriched for the GR half-site motif (TGTYCY/RGRACA) ${ }^{14}$ (Supplementary Fig. 2).

STAT5 and NFIB binding at the Csn $1 s 2 b$ DE do not overlap (Figs. 1d and 2a), suggesting the possibility of their distinct contributions in establishing a functional enhancer. Conversely, STAT5 and NFIB binding coincides at the Csn1s2a candidate regulatory regions (Fig. 1c). The presence of H3K4me1 marks in the candidate Csn $1 s 2 b$ DE supports its status as enhancer. STAT5 binding at a GAS motif within intron 9 of the $C s n 1 s 2 b$ gene was detected during pregnancy but it sharply declined during lactation (Fig. 1b and Supplementary Fig. 1b) suggesting that it might activate the locus during pregnancy.

Identification of TF building blocks required for the establishment and function of the distal enhancer. Next, we explored the biological significance of the $\operatorname{Csn} 1 s 2 b \mathrm{DE}$ and its individual building blocks through the introduction of mutations into the mouse genome (Supplementary Tables 2 and 3). We addressed the potential function of the two canonical GAS motifs (TTCnnnGAA) recognized by STAT5 (sites S1 and S2) and the NFIB motif (TGGCA) (N), all of which align with the respective ChIP-seq peaks (Fig. 2a). A non-canonical GAS motif (S3) with a 4 bp spacer (TTCnnnnGAA) was detected between the NFIB site and the STAT5 site S1. Such non-canonical GAS motifs are known to be recognized by STAT6. We generated mice carrying individual or combinatorial mutations disrupting the GAS and NFIB motifs (Fig. 2a). Although the deletion of a single $T$ from the S2 GAS motif $(\Delta \mathrm{S} 2)$ (Supplementary Table 3) led to an insignificant reduction of Csn1s $2 b$ expression (Fig. 2b), it nevertheless resulted in an $\sim 40 \%$ loss of STAT5 binding (Fig. 2c, d), suggesting a compensatory role of STAT5 binding to site S1. The Wap and Cish genes were used as ChIP-seq controls (Supplementary Fig. 3a). Disruption of the NFIB motif $(\Delta \mathrm{N})$ was accomplished with a $15 \mathrm{bp}$ deletion that removed the ' $\mathrm{A}$ ' from the canonical TGGCA motif (Supplementary Table 3). Csn1s2b expression was overtly unaffected, as was NFIB and STAT5 binding at the DE (Fig. 2b, c). Although the mutated site (TGGCT) does not match known NFIB binding sites, we cannot rule out the possibility that this site, in conjunction with intact STAT5 sites facilitates NFIB binding. To further address this issue, we introduced a $14 \mathrm{bp}$ deletion spanning the entire NFIB site into the $\Delta \mathrm{S} 2$ background $(\Delta \mathrm{N} / \mathrm{S} 2)$. Csn $1 s 2 b$ expression was reduced by $\sim 45 \%$ (Fig. $2 \mathrm{~b}$ ) and coincided with greatly reduced STAT5 binding and diminished H3K27ac marks (Fig. 2c, d). The STAT5 and NFIB coverage in the Csn $12 b$ enhancer of mutants $(\Delta \mathrm{S} 2, \Delta \mathrm{N}$ and $\Delta \mathrm{N} / \mathrm{S} 2$ ) was confirmed by the raw read mapping (Supplementary Fig. 3b), demonstrating that STAT5 and NFIB are still bound to the mutant enhancer. GR binding was reduced in the $\Delta$ S2 mutant (Supplementary Figure 4) suggesting either cooperativity between these sites or tethering of GR to STAT5.

Importance of the non-canonical GAS motif in Csn1s2b gene expression. To address the possibility of additional TF binding sites in the DE, we dug deeper and analyzed the remaining sequences under the ChIP-seq peaks. First, we introduced a deletion spanning the NFIB site and the non-canonical GAS 
a

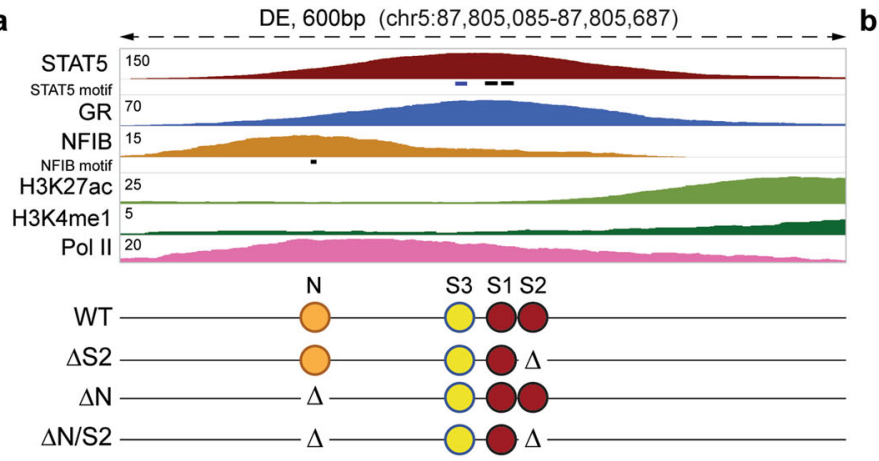

b

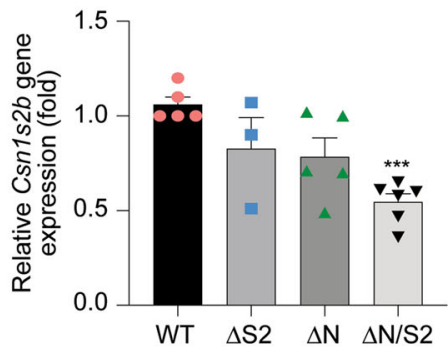

d

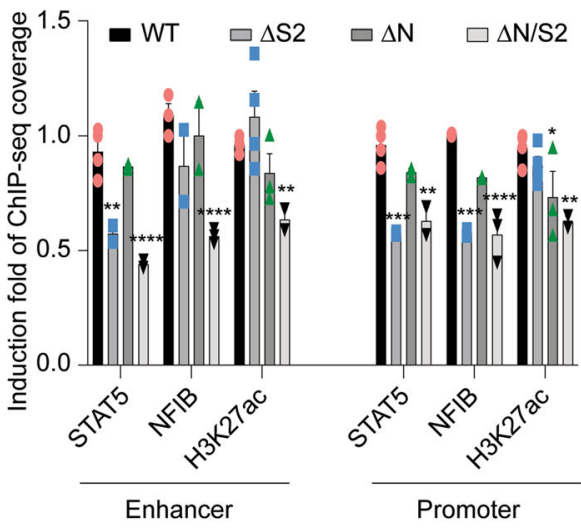

Fig. 2 Redundant and non-redundant functions of STAT5 and NFIB sites in the Csn1s2b distal enhancer. a Genomic feature of the Csn1s $2 b$ distal enhancer (DE) and diagram of the deletions introduced in the mouse genome using CRISPR/Cas9 genome editing. TF binding sites were mutated individually $(\Delta \mathrm{S} 2$ and $\Delta \mathrm{N})$ or in combination ( $\Delta \mathrm{N} / \mathrm{S} 2$ ). While S1 and S2 display canonical GAS motifs (burgundy circles), S3 (yellow circle) is a noncanonical sequence with a 4 bb spacer. N (orange circle) is a NFIB binding site. b Csn1s $2 b$ mRNA levels in day 10 lactating (L10) mammary tissues from WT and mutant mice were measured by GRT-PCR and normalized to Gapdh levels. Results are shown as the means \pm s.e.m. of independent biological replicates (WT and $\Delta \mathrm{N}, n=5 ; \Delta \mathrm{S} 2$ and $\Delta \mathrm{N} / \mathrm{S} 2, n=6$ ). One-way ANOVA followed by Dunnett's multiple comparisons test was used to evaluate the statistical significance of differences between WT and each mutant mouse line. $p$-value $=0.21,0.63$ and 0.0006 , respectively. c The Csn1s $2 b$ locus was profiled using ChIP-seq data of WT and mutant tissue. d STAT5, NFIB and H3K27ac coverage was calculated after variation between data set was normalized with Cish promoter coverage. Results are shown as the means \pm s.e.m. of independent biological replicates (STAT5, H3K27ac of WT and H3K27 of $\Delta$ S2, $n=4$; NFIB of WT, H3K27ac of $\Delta \mathrm{N}$ and NFIB of $\Delta \mathrm{N} / \mathrm{S} 2, n=3$; STAT5, NFIB of $\Delta \mathrm{S} 2$ and $\Delta \mathrm{N}$, STAT5 and H3K27ac of $\Delta \mathrm{N} / \mathrm{S} 2, n=2$ ).

motif S3 ( $\Delta \mathrm{N} / \mathrm{S} 3)$ (Fig. 3a and Supplementary Table 3). Csn1s2b mRNA levels declined by $86 \%$, which paralleled a more than $70 \%$ reduction of STAT5 occupancy and H3K27ac marks at the DE and promoter (Fig. 3b-d). Cish and Wap were used as ChIP-seq controls (Supplementary Fig. 5). To determine whether sites S1 and S2 foster the residual enhancer activity, we introduced a deletion spanning S1 and S3 (Fig. 3a and Supplementary Table 3). In addition, as a result of imperfect CRISPR/Cas9 genome editing $^{15}$, the NFIB site was disrupted. Csn1s $2 b$ mRNA levels in this mutant $(\Delta \mathrm{N} / \mathrm{S} 1 / 3)$ were reduced by $\sim 89 \%$ (Fig. $3 \mathrm{~b}$ ) and the remaining GAS motif S2 is sufficient for residual STAT5 binding (Fig. 3c, d). Lastly, we generated mice carrying a deletion spanning site $\mathrm{S} 3$ and the point mutation in $\mathrm{S} 2(\Delta \mathrm{S} 2 / 3)$ (Fig. 3a). Csn $1 s 2 b$ expression levels were reduced by more than $95 \%$ (Fig. 3b) coinciding with a complete absence of STAT5 and NFIB binding, despite an intact NFIB DNA binding motif (Fig. 3c, d). Similarly, no GR binding was detected despite the presence of an intact GR half-site (Supplementary Fig. 4). The reduction of $\mathrm{H} 3 \mathrm{~K} 27 \mathrm{ac}$ marks coincided with reduced gene expression (Fig. 3c, d and Supplementary Fig. 5). The combined absence of sites $\mathrm{S} 2$ and $\mathrm{S} 3$ resulted in a complete absence of TF binding at the distal enhancer and also in a sharp reduction at the promoter proximal site (Fig. 3c, d), in agreement with the almost complete loss of Csn1s $2 b$ expression. The STAT5 and NFIB coverage and H3K27ac marks at the Csn1s2b locus in wt and mutant tissues are shown in Supplementary Fig. 6. These results provide evidence that the non-canonical STAT5 binding motif, and possibly the surrounding sequences, is a key element in the $\mathrm{DE}$ and synergizes with the canonical site S2. The integration of the results from all of the mutants strongly suggests that STAT5 preferentially binds at the non-canonical site S3. Ultimate proof for this conclusion would require the specific deletion of S3, which we did not accomplish in this study.

Temporal activity of the Csn1s2b intronic enhancer. Our ChIPseq data had revealed a putative enhancer in intron 9 of the $C s n 1 s 2 b$ gene (Fig. $1 \mathrm{~b}$ and Supplementary Fig. 1a-b). Like other mammary candidate enhancers, it was bound by STAT5, NFIB, GR, MED1 and Pol II and coincided with activating histone marks H3K27ac and H3K4me1 (Fig. 1d). STAT5 binding was prominent during pregnancy and declined during lactation (Fig. 4a), suggesting the possibility of a priming function in the activation of the Csn1s2b locus. To test this hypothesis, we generated mice with two distinct deletions targeting the GAS and NFIB motifs. The GAS motif was disrupted through the introduction of either a $3 \mathrm{bp}$ or $14 \mathrm{bp}$ deletion $(\Delta \mathrm{IE}-\mathrm{S})$ and a $36 \mathrm{bp}$ 


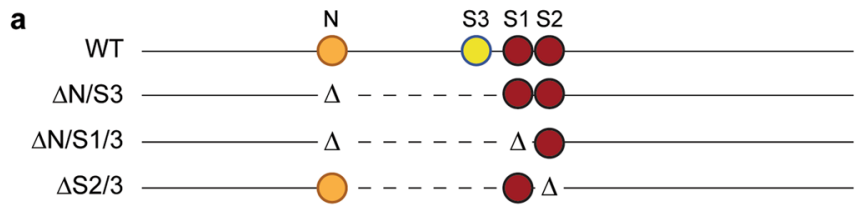

b
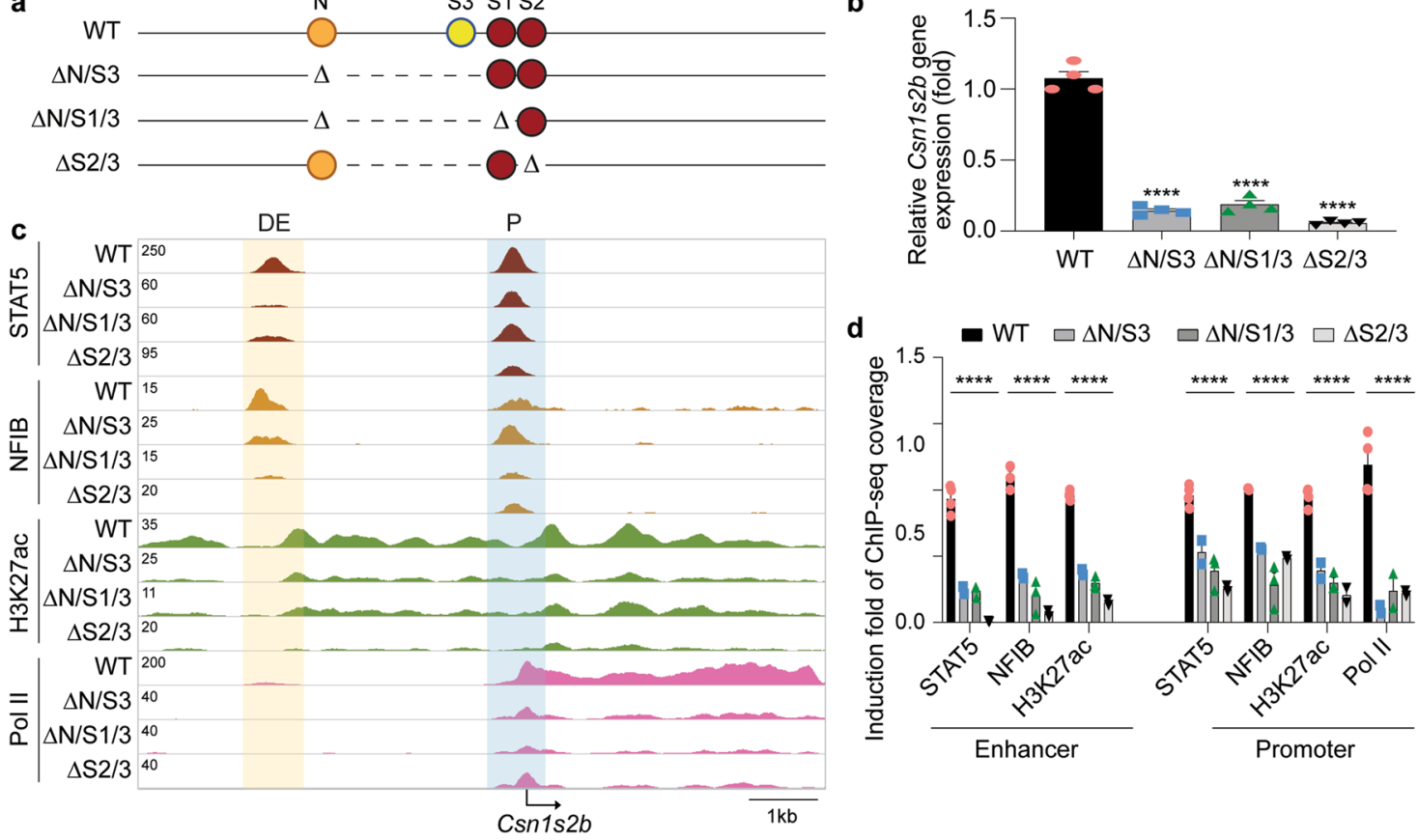

Fig. 3 Requirement of the non-canonical STAT5 site in the Csn1s2b distal enhancer. a Diagram of the enhancer deletions introduced using CRISPR/Cas 9 genome editing. The non-canonical STAT5 motif was deleted in combination with other enhancer motifs ( $\Delta N / S 3, \Delta N / S 1 / 3$ and $\Delta S 2 / 3)$. The canonical GAS motifs S1 and S2 are shown as burgundy circles, and the non-canonical GAS motif S3 is shown in yellow. NFIB binding site is shown in orange. b Csn1s2b mRNA levels were measured by qRT-PCR in day 10 lactating (L10) mammary tissue of WT and mutant mice and normalized to Gapdh levels. Results are shown as the means \pm s.e.m. of independent biological replicates (WT, $n=5 ; \Delta N / S 3, \Delta N / S 1 / 3$ and $\Delta S 2 / 3, n=4$ ). One-way ANOVA followed by Dunnett's multiple comparisons test was used to evaluate the statistical significance of differences between WT and each mutant mouse. $p$-value $=0.0001,0.0001$ and 0.0001, respectively. c The consequences of enhancer deletions were confirmed by STAT5A, NFIB, H3K27ac and Pol II ChIP-seq analysis in WT and mutant tissues at L10. The Cish locus served as control (Supplementary Fig. 3). d STAT5, NFIB, H3K27ac and Pol II coverage was calculated after variation between data set was normalized by Cish promoter coverage. Results are shown as the means \pm s.e.m. of independent biological replicates $(n=2$ to 4$)$.

deletion covered the GAS and NFIB motifs ( $\triangle \mathrm{IE})$ (Fig. 4a and Supplementary Table 3). As expected, the combined deletion of the STAT5 and NFIB binding sites ( $\triangle \mathrm{IE})$ abrogated STAT5 binding, H3K27ac and Pol II coverage at L1 (Fig. 4b). Residual NFIB binding suggests that this TFs might bind indirectly to chromatin and not through its core DNA motif. STAT5 binding was also diminished at the $\mathrm{DE}$ and promoter region, indicating a functional role of the intronic enhancer. The Cish gene was used as a ChIP-seq control (Fig. 4b). Deletion of the GAS site by itself $(\triangle \mathrm{IE}-\mathrm{S})$ or in combination with the NFIB motif $(\triangle \mathrm{IE})$ resulted in a reduction of Csn $1 s 2 b$ mRNA levels at L1 by $\sim 50 \%$ and $80 \%$, respectively (Fig. 4c). In contrast, at L10, Csn1s $2 b$ mRNA levels were not significantly reduced, in agreement with the absence of enhancer structures in wt mammary gland tissue. In accordance with the expression data, the $\triangle \mathrm{IE}$ mutation impacted STAT5 binding and $\mathrm{H} 3 \mathrm{~K} 27 \mathrm{ac}$ at the promoter at L1, but not so at L10 (Fig. 4d).

A 3' super-enhancer activates Csn1s2b expression. Our genetic analyses demonstrated that the distal enhancer is a key driver of $C s n 1 s 2 b$ expression throughout lactation and the intronic enhancer is most prominent at the intersection of pregnancy and early lactation. While there are no additional overt enhancer marks between the body of the Csn $1 s 2 b$ gene and the neighboring Csn1s2a and Prr27 genes, we explored the possibility of further enhancers and monitored activating chromatin marks in the extended casein locus at L1 (Fig. 5a). A $10 \mathrm{~kb}$ sequence highly enriched with H3K27ac and H3K4me1 marks was identified 3' of the Prr27 gene, $65 \mathrm{~kb} 3$ ' of the Csn1s2b gene (Fig. 5a). ChIP-seq identified STAT5, NFIB, GR and MED1 binding to several sites in this region and the Rose algorithm called it a super-enhancer (SE). Expression of the Prr27 gene, which is located between the Csn1s2b gene and the SE, is barely detectable in mammary tissue, suggesting that it is not a genuine target of this candidate SE. 3C analyses demonstrated that this SE interacted with the neighboring $C s n 1 s 2 b$ and $C s n 3$ genes (Fig. 5b). Deletion of the SE from the mouse genome resulted in a more than $90 \%$ reduction of Csn1s $2 b$ mRNA at p18 (Fig. 5c) suggesting its pivotal role in gene activation during pregnancy. Expression of the Csn1s2a gene was reduced by $\sim 40 \%$, which was statistically not significant. Since mice lacking the SE failed to nurse their pups, it was not possible to investigate $C s n 1 s 2 b$ expression during lactation. Failure to lactate is not the result of reduced expression of $C s n 1 s 2 b$ since mice lacking the distal enhancer can lactate despite even lower $C s n 1 s 2 b$ expression levels. To identify the cause of lactation failure, further research is needed.

Deletion of the SE resulted in the complete loss of H3K27c marks and transcription factor binding at that region (Fig. 5d). Importantly, the absence of significant H3K27ac marks in the $C s n 1 s 2 b$ promoter and the distal (DE) and intronic enhancers (IE) is reflective of loss of gene expression. Notably, deletion of the SE severely impacted STAT5 binding at the Csn $1 s 2 b$ promoter but less so at the DE (Fig. 5d). Conversely, STAT5 binding at the intronic enhancer was elevated. The Csn1s2a locus served as a ChIP-seq control. These findings strongly suggest a dominant function of the SE in activating the $C s n 1 s 2 b$ promoter and that the distal and intronic enhancers cannot function independently and compensate for the absence of the SE at the transition of pregnancy to lactation (p18 and L1). 

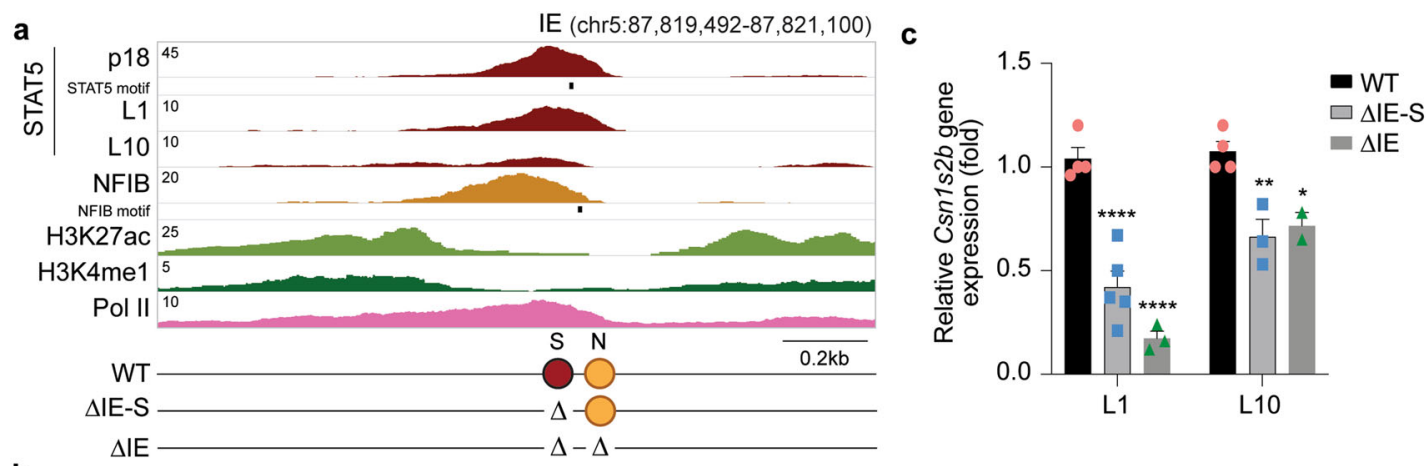

b
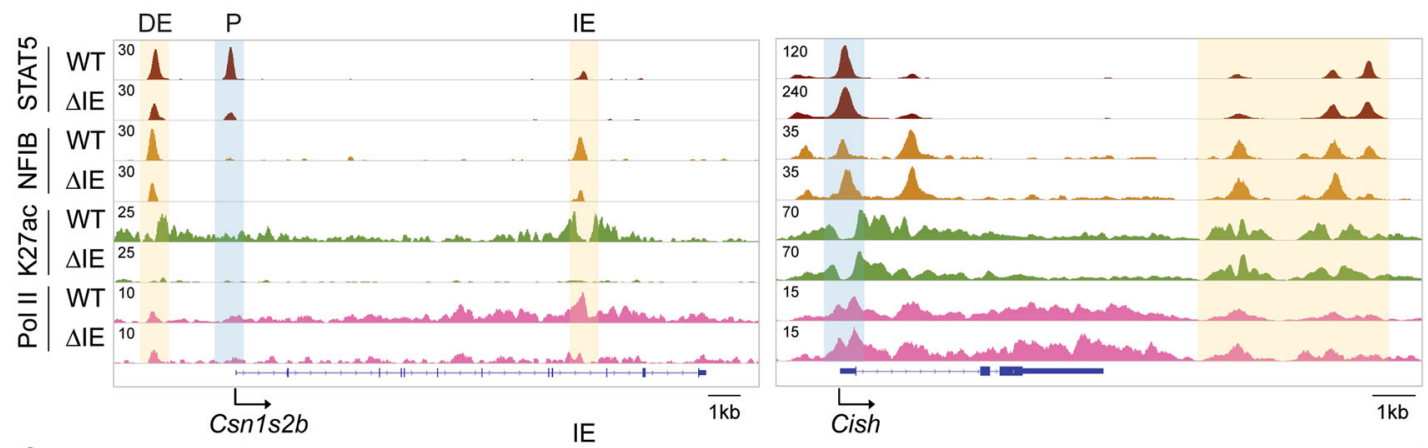

d
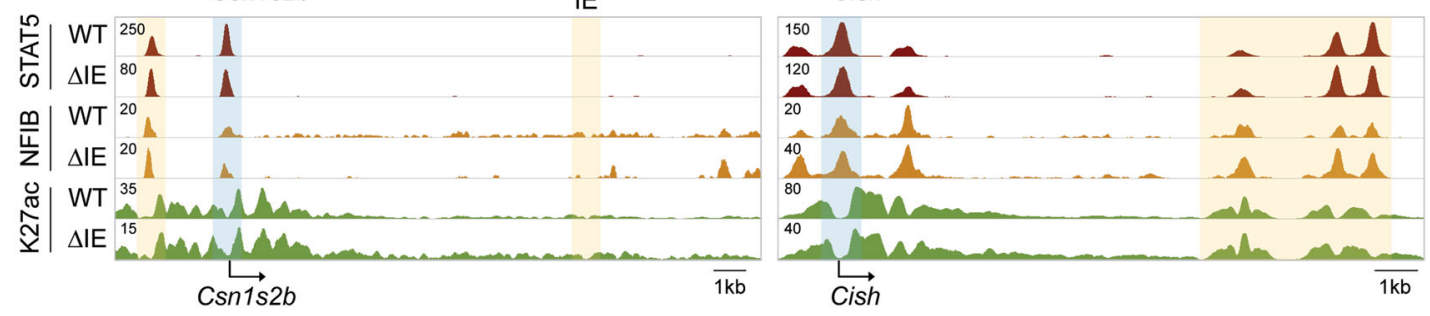

Fig. 4 The intronic enhancer controls Csn1s2b expression in early lactation. a Genomic feature of the Csn1s2b locus in mammary tissue at day one of lactation (L1) and diagram of mutant mice. $\Delta \mathrm{IE}-\mathrm{S}$, deletion of the GAS motif; $\Delta \mathrm{IE}$, deletion of the entire intronic enhancer. $\mathbf{b}$ and $\mathbf{d}$ TF binding, H3K27ac marks and Pol II loading at the Csn1s2b locus in WT and mutant tissue at L1 (b) and L10 (d). The Cish locus served as a ChIP-seq control. c Csn1s2b mRNA levels were measured by qRT-PCR in L1 and L10 mammary tissue from WT and $\Delta \mathrm{IE}$ mutant mice and normalized to Gapdh levels. Results are shown as the means \pm s.e.m. of independent biological replicates (WT of L1 and L10, $n=4$; L1 of $\Delta \mathrm{IE}-\mathrm{S}, n=5$; L10 of $\Delta \mathrm{IE}-\mathrm{S}$, and L1 and L10 of $\Delta \mathrm{IE}, n=3$ ). Two-way ANOVA followed by Tukey's multiple comparisons test was used to evaluate the statistical significance of differences. $p$-value $=0.0001,0.0001,0.0076$ and 0.0478 , respectively.

Promoter activity is modulated by NFIB. In addition to the distal and intronic enhancers, and the downstream SE, STAT5 and NFIB binding was also recorded in the promoter region within $100 \mathrm{bp}$ of the TSS (Fig. 6a). We introduced an 18 bp deletion into the mouse genome leading to the loss of the NFIB site (Supplementary Table 3). Csn $1 s 2 b$ expression was reduced by $\sim 60 \%$ (Fig. $6 \mathrm{~b}$ ), which coincided with reduced $\mathrm{H} 3 \mathrm{~K} 27 \mathrm{ac}$ marks and Pol II coverage (Fig. 6c). Residual NFIB coverage could be the result of indirect binding through STAT5.

\section{Discussion}

Here we use experimental mouse genetics and functionally investigate two mammary-gland enhancers and one superenhancer that distinctly control expression of the $C s n 1 s 2 b$ gene during pregnancy and lactation. A distal enhancer preferentially controls gene expression throughout lactation, an intronic enhancer is active in early lactation and a super-enhancer is needed for the activation of the locus during pregnancy (Fig. 7). We also gained new insight into the architecture and biology of redundant and non-redundant enhancer building blocks based on the mammary gland-enriched transcription factors STAT5 and NFIB.

Unlike most mammary gland enhancers, which are activated during early pregnancy and induce gene expression prior to lactation 6,16 , the $C s n 1 s 2 b$ enhancer is preferentially established during lactation where it contributes to a several hundred fold expression induction. While most, if not all, mammary gland enhancers employ the cytokine-activated TF STAT5 as a core building block together with NFIB and GR, differential temporal recruitment has been observed. Although the underlying logic for a temporally distinct activation of seemingly identical enhancer sequences at different stages during mammary development, i.e. pregnancy versus lactation, it is not clear that the respective TF binding sites might have different affinities. In support of this, genetic studies in mammary tissue have revealed that the concentration of STAT5 can influence gene activation patterns ${ }^{6,17}$. Alternatively, seemingly identical regulatory elements might become gradually accessible during differentiation, as shown in the $a$-globin locus ${ }^{18}$. The Csn $1 s 2 b$ distal enhancer is overtly more complex than other mammary enhancers ${ }^{19}$ and contains two canonical and one non-canonical STAT5 binding sites in addition to an NFIB site and a GR half-site, which could contribute to its lactation-restricted activation.

Unlike other genetically validated enhancers where STAT5 binds to a canonical GAS motif ${ }^{19-22}$, the $\operatorname{Csn} 1 s 2 b$ enhancer contains a functional non-canonical GAS motif in addition to two canonical sites. The contribution of canonical and non-canonical TF binding sites within enhancers is still being debated and it 

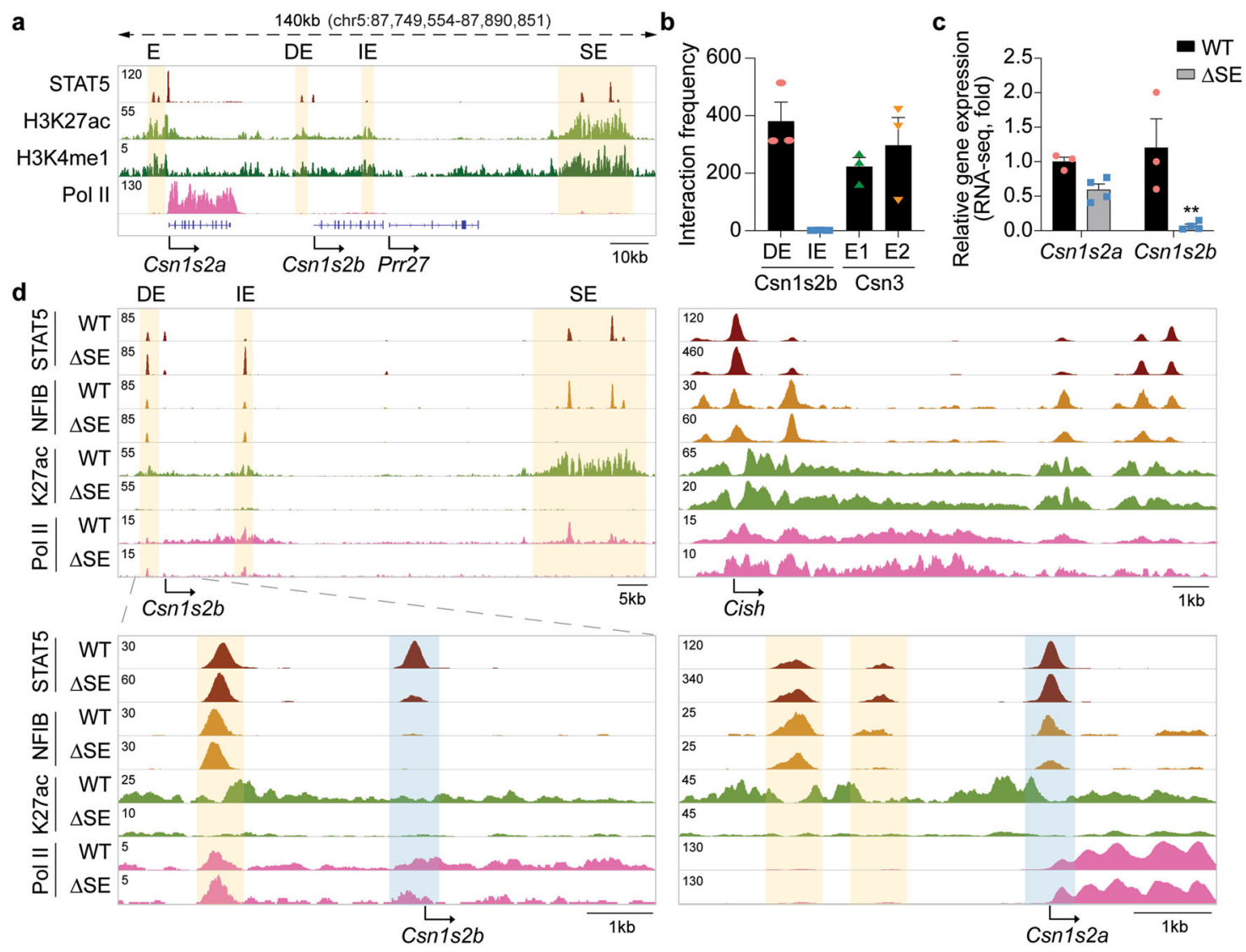

Fig. 5 A super-enhancer activates Csn1s2b expression during pregnancy. a Genomic feature of the Csn1s2a/b locus, including the downstream Prr27 gene and the super-enhancer (SE) in L1 mammary tissue. b Interactions between SE and the regulatory elements of Csn1s $2 b$ and Csn3 were detected by $3 \mathrm{C}$ analysis. c Csn1s2a/b mRNA levels were measured by RNA-seq at day 18 of pregnancy (p18) in WT and $\Delta$ SE (deletion of the $10 \mathrm{~kb}$ SE) mice. Results are shown as the means \pm s.e.m. of independent biological replicates $(n=4)$. Two-way ANOVA followed by Tukey's multiple comparisons test was used to evaluate the statistical significance of differences. Results are shown as the means \pm s.e.m. of independent biological replicates $(n=3)$. $p$-value $=0.28$ and 0.003 d STAT5 and NFIB binding, H3K27ac marks and Pol II coverage in $\Delta$ SE mammary tissue at L1. The Cish locus served as control.

might depend on the specific transcription factor and target tissue. STAT5 exists in two isoforms, STAT5A and STAT5B, which are encoded by two distinct genes ${ }^{23}$. In mammary tissue, STAT5A levels exceed STAT5B 2-3 fold ${ }^{21}$ and STAT5A ChIP-seq experiments during pregnancy and lactation revealed that approximately $90 \%$ of all high-quality peaks coincide with the canonical GAS motif (TTCnnnGAA) ${ }^{16}$. Within mammary enhancers the percentage is even higher. It remains to be determined whether STAT5B recognizes the canonical GAS motif in mammary tissue. In liver STATB levels exceed STAT5A by approximately 10 -fold and the majority of STAT5B ChIP-seq peaks coincide with the classical GAS motif ${ }^{24}$. A similar observation was made in $\mathrm{T}$ cells ${ }^{20}$. While GAS motifs with a $4 \mathrm{bp}$ spacer are generally recognized by STAT6, another STAT member contributing to the differentiation of mammary alveolar cells ${ }^{25}$, this non-canonical site is also recognized by STAT5 in the $C s n 1 s 2 b$ distal enhancer.

Our finding that NFIB, a critical co-activator for a range of mammary genes, including $C s n 1 s 2 b^{26}$, can bind to the enhancer lacking the DNA binding motif adds further intrigue and suggests that the recruitment of multiple TFs can be facilitated through a single anchor, STAT5 in mammary enhancers. Our results also suggest that GR binding to its half-site within the distal enhancer might require the cooperative presence of a neighboring STAT5 site. Alternatively, GR could tether to STAT5 as shown in the Wap gene super-enhancer that contains a STAT5 binding motif but lacks a GR motif ${ }^{19}$. The progesterone receptor (PR) binds to GR motifs ${ }^{27}$ and is required for mammary alveolar development ${ }^{28,29}$. We analyzed PR ChIP-seq data from mammary tissue from progesterone treated non-parous mice ${ }^{30}$ and no binding was detected at the Csn $1 s 2 b$ distal enhancer with a PR half-site motif. However, conclusions from these experiments are limited since they were conducted in non-parous mice that lack the differentiated alveolar compartment. Similarly, ChIP-seq data from the estrogen receptor (ER) did not reveal any binding ${ }^{31}$. It is conceivable that the presence of four TF binding motifs in the distal enhancer region permits additional TFs to bind through less conserved DNA binding sites. The presence of four TF binding blocks in the distal enhancer, possibly in synergy with additional promoter and intronic elements, enables high Csn $1 s 2 b$ expression levels during lactation. It remains to be elucidated why overtly equivalent enhancers activate other casein genes already during pregnancy. Of note, the more than $95 \%$ reduction of Csn1s $2 b$ expression caused by the deletion of the distal enhancer had no overt impact on lactation and is in agreement with other species that lack a functional $C s n 1 s 2 b$ gene.

STAT5, GR and NFIB jointly occupy candidate enhancers of 'mammary-specific' genes $6,8,16$ and their contributions in activating these genes during pregnancy and lactation have been investigated in mutant mice lacking these TFs. Since the global deletion of transcription factors can have widespread consequences on a given cell, such experiments may have limited impact on understanding their role on specific genes. As such, proliferation and differentiation of mammary epithelium during 

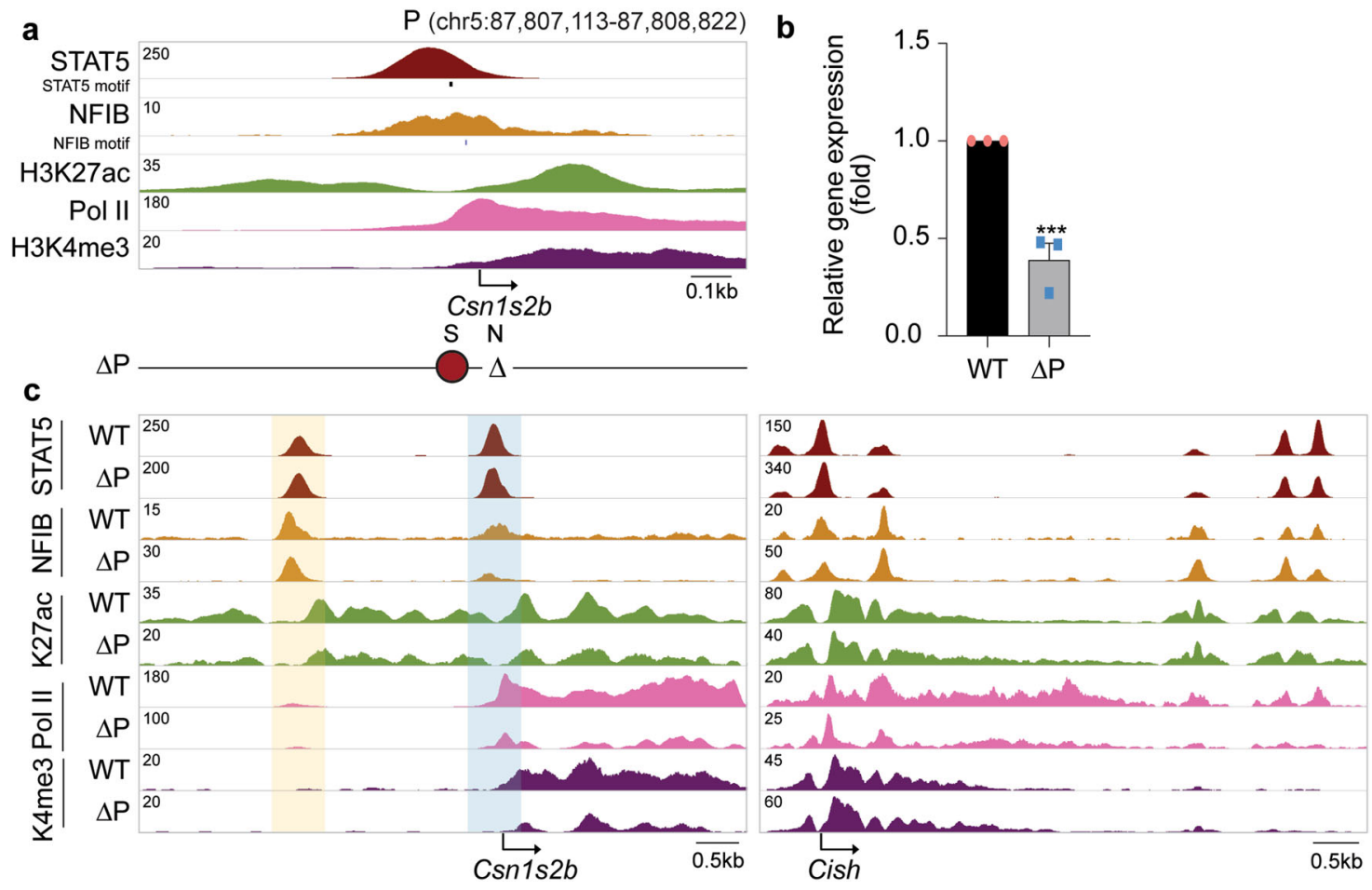

Fig. 6 The NFIB site in the Csn1s2 $\mathbf{b}$ promoter modulates gene activity. a Genomic feature of the Csn1s $2 b$ promoter region in mammary tissue at day ten of lactation. $\Delta \mathrm{P}$, deletion of the NFIB motif. $\mathbf{b}$ mRNA levels of Csn1s $2 b$ gene were measured by qRT-PCR in L1 mammary tissue from WT and $\Delta \mathrm{P}$ mutant mice and normalized to Gapdh levels. Results are shown as the means \pm s.e.m. of independent biological replicates $(n=3)$. Unpaired two-tailed t-test was used to evaluate the statistical significance of differences. $p$-value $=0.002$ c The consequences of super-enhancer deletion were confirmed by ChIP-seq analysis in WT and mutant tissues at L10. The Cish locus served as a ChIP-seq control.

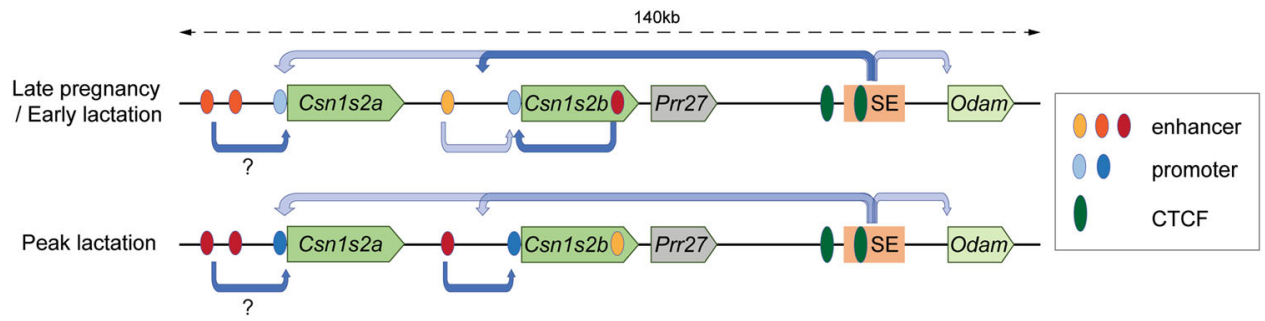

Fig. 7 Proposed model of the activation of the Csn1s2 $\mathbf{a} / \mathbf{b}$ locus during pregnancy and lactation. The Csn1s $2 b$ gene is under the control of three distinct enhancers; a distal enhancer controlling gene expression throughout lactation, an intronic enhancer active at late pregnancy and early lactation, and a super-enhancer (SE) required for the activation of the Csn1s2b locus. While the SE has limited influence on the expression of the Csn1s2a and Odam genes, it does not activate the Prr27 gene in mammary tissue. Two candidate enhancers have been identified in the Csn1s2a distal region, but their function has not been validated through experimental genetics. The color intensity of promoter and enhancer elements at late pregnancy and peak lactation reflects their relative strengths. Darker colors indicate increased strength. Candidate Csn1s2a enhancer and promoter elements are shown but their biological strength is not known. CTCF binding sites have been identified ${ }^{38}$.

pregnancy is greatly impaired in the combined absence of Stat5a and Stat $5 b$, thus making it impossible to define their gene-specific roles. Targeted deletions of three STAT5 sites, in the mouse Wap locus, individually or in combination, defined redundant and non-redundant functions of an enhancer structure essential for gene activation during pregnancy ${ }^{19}$. In a classical study, Burdon and colleagues $^{32}$ employed transgenic mice to explore three STAT5 binding sites in a sheep $\beta$-lactoglobulin transgene ${ }^{32}$ and suggested some degree of cooperativity between canonical and non-canonical STAT5 binding sites. However, not all genes under the control of STAT5 rely on cooperativity ${ }^{20-22}$. A role for the GR in mammary gene regulation is not that clear. Mice lacking the $\mathrm{GR}^{33}$ or expressing a GR devoid of its DNA binding domain ${ }^{34}$ displayed a slightly impaired development of mammary ducts in non-parous mice. However, alveolar differentiation during pregnancy and expression of milk proteins during lactation were unimpaired, demonstrating that the GR is not required for normal mammary function and a potential compensatory function of the mineralocorticoid receptor has been proposed ${ }^{33}$. In contrast to the GR, the presence of the PR is required for the outgrowth and branching of mammary ducts ${ }^{28,35}$. However, since mice lacking the PR are infertile, mammary development and function during pregnancy has not been investigated. Moreover, PR ChIPseq data from lactating mammary tissue are not available. However, studies using mouse mammary cell lines have revealed important functions of the $\mathrm{PR}$ in the regulation of the Csn2 gene ${ }^{36}$. NFIB isoforms are abundantly expressed in mammary tissue $^{37}$ and deletion of the Nfib gene by itself or in combination with Stat5 supported the concept of cooperativity in gene activation in mammary tissue ${ }^{26}$. 
In addition to the distal enhancer, we identified a superenhancer (SE) essential for the activation of the Csn1s $2 b$. We propose that this SE, which is located next to the Odam gene and separated from $C s n 1 s 2 b$ by the Prr27 gene, was part of the evolutionary older $C s n 3$ gene, and the younger $C s n 1 s 2 b$ gene captured its activity. Csn 3 and its neighboring odontogenic ameloblast-associated (Odam) gene originated from a common precursor $^{9}$ and the gene arrangement in this locus (Prr27, Odam, Fdcsp and Csn3) predates the emergence of Csn1s $2 b$. In contrast to $C s n 1 s 2 b$, pregnancy expression of the neighboring Csn $1 s 2 a$ gene is not overtly controlled by the SE. The molecular mechanism underlying lactation failure in mice lacking the SE needs further investigation and might be the result of deregulation of the entire locus. Two CTCF binding sites associated with this $\mathrm{SE}^{38}$ might be an early signature of the casein locus before its expansion that added four additional casein genes, including $C s n 1 s 2 b$. However, deletion of these CTCF binding sites, did not alter $\operatorname{Csn} 1 s 2 b$ expression ${ }^{38}$, suggesting that they have limited biological activity.

The increasing use of a wide range of ChIP-seq and chromatin capture approaches suggests that the mammalian genome is riddled with candidate enhancers that potentially control the spatio-temporal expression of lineage-specific genes ${ }^{39-43}$. However, as stated by one of the reviewers, we humans are not always good in selecting the TF sites or potential regulators that turn out to be important or essential. As shown here, uncovering the function and complexity of enhancers requires detailed genetic interventions. The casein locus with five genes expressed exclusively in mammary glands, three interspersed genes expressed in salivary glands and at least 20 candidate mammary enhancers, super-enhancers and CTCF sites remains a case study in evolutionary strategies to ensure uncompromised gene regulation. As further genetic inquiries are conducted, the multiplicity of regulatory building blocks controlling mammary- and salivary-gland specificity and cytokine-induced gene activation will continue to unfold.

\section{Methods}

Mice. All animals were housed and handled according to the Guide for the Care and Use of Laboratory Animals (8th edition) and all animal experiments were approved by the Animal Care and Use Committee (ACUC) of National Institute of Diabetes and Digestive and Kidney Diseases (NIDDK, MD) and performed under the NIDDK animal protocol K089-LGP-17. CRISPR/Cas9 targeted mice were generated using C57BL/6 N mice (Charles River) by the transgenic core of the National Heart, Lung, and Blood Institute (NHLBI). Single-guide RNAs (sgRNA) were obtained from either OriGene (Rockville, MD) or Thermo Fisher Scientific (Supplementary Table 2). Target-specific sgRNAs and in vitro transcribed Cas 9 mRNA were co-microinjected into the cytoplasm of fertilized eggs for founder mouse production. The $\Delta \mathrm{N} / \mathrm{S} 2$ and $\Delta \mathrm{S} 2 / 3$ mutant mouse was generated by injecting sgRNAs for NFIB site into zygotes collected from $\Delta \mathrm{S} 2$ mutant mice. All mice were genotyped by PCR amplification and Sanger sequencing (Macrogen and Quintara Biosciences) with genomic DNA from mouse tails (Supplementary Table 3) and only homozygous mutant mice used in the study.

\section{Chromatin immunoprecipitation sequencing (ChIP-seq) and data analysis} Mammary tissues from specific stages during pregnancy and lactation were harvested, and stored at $-80^{\circ} \mathrm{C}$. The frozen-stored tissues were ground into powder in liquid nitrogen. Chromatin was fixed with formaldehyde ( $1 \%$ final concentration) for $15 \mathrm{~min}$ at room temperature, and then quenched with glycine $(0.125 \mathrm{M}$ final concentration). Samples were processed as previously described ${ }^{21}$. The following antibodies were used for ChIP-seq: STAT5A (Santa Cruz Biotechnology, sc271542), GR (Thermo Fisher Scientific, PA1-511A), NFIB (Sigma-Aldrich HPA003956), H3K27ac (Abcam, ab4729), RNA polymerase II (Abcam, ab5408), H3K4mel (Active Motif, 39297) and H3K4me3 (Millipore, 07-473). Libraries for next-generation sequencing were prepared and sequenced with a HiSeq 2500 or 3000 instrument (Illumina)

The raw data were subjected to QC analyses using the FastQC tool (version 0.11.9) (https://www.bioinformatics.babraham.ac.uk/projects/fastqc/). Quality filtering and alignment of the raw reads was done using Trimmomatic ${ }^{44}$ (version 0.36), Bowtie ${ }^{45}$ (version 1.2.2) and Samtools ${ }^{46}$ (version 1.8), with the parameter '-m 1 ' to keep only uniquely mapped reads, using the reference genome mm10. Picard tools (version 2.9.2, Broad Institute. Picard, http://broadinstitute.github.io/picard/ 2016) was used to remove duplicates. Homer ${ }^{47}$ (version 4.8.2) and DeepTools ${ }^{48}$ (version 3.1.3) software was applied to generate bedGraph files, separately. Integrative Genomics Viewer ${ }^{49}$ (version 2.5.3) was used for visualization. Each ChIP-seq experiment was conducted for more than two replicates. DeepTools was used to obtain the Pearson and Spearman correlation between the replicates.

In order to identify regions of ChIP-seq enrichment over the background, MACS $^{50}$ (version 2.2.7.1) peak finding algorithm was used. Peak calling of TFs and histone markers for WT and mutants was done for replicates, which were subsequently overlapped using Bedtools ${ }^{51}$ (version 2.29.2) to identify highconfident peaks. TF bound enhancers were considered as true enhancer elements if they showed H3K27ac underneath. Coverage plots (normalized to 10 million reads) and motif analysis with default settings were done using Homer software.

Coverage plots were generated using Homer software with the bedGraph as input. R and the packages dplyr (https://CRAN.R-project.org/package=dplyr) and ggplot $2^{52}$ were used for visualization. Sequence read numbers were calculated using Samtools software with sorted bam files.

RNA isolation and quantitative real-time PCR (qRT-PCR). Total RNA was extracted from frozen mammary tissue of wild type and mutant mice using a homogenizer and the PureLink RNA Mini kit according to the manufacturer's instructions (Thermo Fisher Scientific). Total RNA $(1 \mu \mathrm{g})$ was reverse transcribed for $50 \mathrm{~min}$ at $50^{\circ} \mathrm{C}$ using $50 \mu \mathrm{M}$ oligo dT and $2 \mu \mathrm{l}$ of SuperScript III (Thermo Fisher Scientific) in a $20 \mu \mathrm{l}$ reaction. Quantitative real-time PCR (qRT-PCR) was performed using TaqMan probes (Csn1s2a, Mm00839343_m1; Csn1s2b,

Mm00839674_m1; mouse Gapdh, Mm99999915_g1, Thermo Fisher scientific) on the CFX384 Real-Time PCR Detection System (Bio-Rad) according to the manufacturer's instructions. PCR conditions were $95^{\circ} \mathrm{C}$ for $30 \mathrm{~s}, 95^{\circ} \mathrm{C}$ for $15 \mathrm{~s}$, and $60^{\circ} \mathrm{C}$ for $30 \mathrm{~s}$ for 40 cycles. All reactions were done in triplicate and normalized to the housekeeping gene Gapdh. Relative differences in PCR results were calculated using the comparative cycle threshold $\left(C_{T}\right)$ method.

Total RNA-seq analysis. The frozen-stored tissues were ground into powder in liquid nitrogen and Total RNA was extracted using the PureLink RNA Mini kit according to the manufacturer's instructions (Thermo Fisher Scientific). Ribosomal RNA was removed from $1 \mu \mathrm{g}$ of total RNAs and cDNA was synthesized using SuperScript III (Invitrogen). Libraries for sequencing were prepared according to the manufacturer's instructions with TruSeq Stranded Total RNA Library Prep Kit with Ribo-Zero Gold (Illumina, RS-122-2301) and 50 bp paired-end sequencing was done with a HiSeq 2500 instrument (Illumina).

The raw data were subjected to QC analyses using the FastQC tool (version 0.11.9) (https://www.bioinformatics.babraham.ac.uk/projects/fastqc/). Total RNAseq read quality control was done using Trimmomatic ${ }^{44}$ (version 0.36 ) and STAR RNA-seq ${ }^{53}$ (version 2.5.4a) using 50 bp paired-end mode was used to align the reads (mm10). HTSeq ${ }^{54}$ (version 0.9.1) was to retrieve the raw counts and subsequently, R (version 3.6.3) (https://www.R-project.org/), Bioconductor (version 3.10$)^{55}$ and DESeq $2^{52}$ were used. Additionally, the RUVSeq ${ }^{56}$ package was applied to remove confounding factors. The data were pre-filtered keeping only those genes, which have at least ten reads in total. The visualization was done using dplyr (https://CRAN.R-project.org/package $=$ dplyr) and ggplot $2^{57}$.

Chromosome conformation capture (3 C). DNA samples for $4 \mathrm{C}$-seq from our previous study ${ }^{58}$ were analyzed by qRT-PCR using SYBR green supermix (Biorad) on the CFX384 Real-Time PCR Detection System (Bio-Rad). The primers used were SE $5^{\prime}$-GTACTCTGGAAAAGTAGGCAGTGC-3', Csn1s2b-DE 5'-AGCTGG CCAACACAAAAGAATGGC-3', Csn1s2b-IE 5' - AGCCAGGTGAGTGAGCTAT GTTC-3', Csn3-E1 5'- GAGTCTAACCACGCTACAGCTTC-3', and Csn3-E2 5'GTAGCTACTTCGGAAACCATCAAGG-3'. Interaction frequencies were normalized to the values of an internal control.

Statistical analyses. For comparison of samples, data were presented as standard deviation in each group and were evaluated with a one-way ANOVA followed by Dunnett's multiple comparisons test, 2-way ANOVA followed by Tukey's multiple comparisons test for comparisons or unpaired two-tailed t-test between WT and mutants using GraphPad Prism 8 (version 8.2.0). A value of $* P<0.05,{ }^{*} * P<0.001$ $* * * P<0.0001, * * * * P<0.00001$ was considered statistically significant. Significances for homer de novo motifs were evaluated with Poisson distribution ${ }^{59}$.

Reporting summary. Further information on research design is available in the Nature Research Reporting Summary linked to this article.

\section{Data availability}

The source data files were obtained or uploaded to Gene Expression Omnibus (GEO). ChIP-seq data of wild-type tissue at L1 and L10 were obtained under GSE74826 ${ }^{19}$, GSE115370 ${ }^{8}$, GSE145193 ${ }^{60}$, GSE127144 and GSE145193 ${ }^{60}$. RNA-seq data for WT at p18, L1 and L10 were downloaded from GSE127140 and GSE115370 . The ChIP-seq and RNA-seq data from WT and mutant mice were uploaded in GSE161620. All files were summarized in Supplementary Data 1 and aligned to reference genome mm10. 
Received: 28 May 2020; Accepted: 16 March 2021;

Published online: 14 April 2021

\section{References}

1. Ong, C. T. \& Corces, V. G. Enhancer function: new insights into the regulation of tissue-specific gene expression. Nat. Rev. Genet 12, 283-293 (2011).

2. Andersson, R. \& Sandelin, A. Determinants of enhancer and promoter activities of regulatory elements. Nat. Rev. Genet 21, 71-87 (2020).

3. Cui, Y. et al. Inactivation of Stat5 in mouse mammary epithelium during pregnancy reveals distinct functions in cell proliferation, survival, and differentiation. Mol. Cell Biol. 24, 8037-8047 (2004).

4. Liu, X. et al. Stat5a is mandatory for adult mammary gland development and lactogenesis. Genes Dev. 11, 179-186 (1997).

5. Shillingford, J. M. et al. Jak2 is an essential tyrosine kinase involved in pregnancy-mediated development of mammary secretory epithelium. Mol. Endocrinol. 16, 563-570 (2002).

6. Yamaji, D., Kang, K., Robinson, G. W. \& Hennighausen, L. Sequential activation of genetic programs in mouse mammary epithelium during pregnancy depends on STAT5A/B concentration. Nucleic Acids Res. 41 1622-1636 (2013).

7. Hennighausen, L. G. \& Sippel, A. E. Characterization and cloning of the mRNAs specific for the lactating mouse mammary gland. Eur. J. Biochem. 125, 131-141 (1982).

8. Lee, H. K., Willi, M., Shin, H. Y., Liu, C. \& Hennighausen, L. Progressing super-enhancer landscape during mammary differentiation controls tissuespecific gene regulation. Nucleic Acids Res. 46, 10796-10809 (2018).

9. Kawasaki, K., Lafont, A. G. \& Sire, J. Y. The evolution of milk casein genes from tooth genes before the origin of mammals. Mol. Biol. Evol. 28, 2053-2061 (2011).

10. Groenen, M. A., Dijkhof, R. J., Verstege, A. J. \& van der Poel, J. J. The complete sequence of the gene encoding bovine alpha s2-casein. Gene $\mathbf{1 2 3}$ 187-193 (1993).

11. Rijnkels, M., Elnitski, L., Miller, W. \& Rosen, J. M. Multispecies comparative analysis of a mammalian-specific genomic domain encoding secretory proteins. Genomics 82, 417-432 (2003).

12. Kuo, T. et al. Genome-wide analysis of glucocorticoid receptor-binding sites in myotubes identifies gene networks modulating insulin signaling. Proc. Natl Acad. Sci. USA 109, 11160-11165 (2012).

13. Rivers, C. A. et al. Glucocorticoid receptor-tethered mineralocorticoid receptors increase glucocorticoid-induced transcriptional responses. Endocrinology 160, 1044-1056 (2019).

14. Cohen, D. M. \& Steger, D. J. Nuclear receptor function through genomics: lessons from the glucocorticoid receptor. Trends Endocrinol. Metab. 28, 531-540 (2017).

15. Shin, H. Y. et al. CRISPR/Cas9 targeting events cause complex deletions and insertions at 17 sites in the mouse genome. Nat. Commun. 8, 15464 (2017).

16. Kang, K., Yamaji, D., Yoo, K. H., Robinson, G. W. \& Hennighausen, L. Mammary-specific gene activation is defined by progressive recruitment of STAT5 during pregnancy and the establishment of H3K4me3 marks. Mol. Cell Biol. 34, 464-473 (2014).

17. Willi, M., Yoo, K. H., Wang, C., Trajanoski, Z. \& Hennighausen, L. Differential cytokine sensitivities of STAT5-dependent enhancers rely on Stat5 autoregulation. Nucleic Acids Res. 44, 10277-10291 (2016).

18. Oudelaar, A. M. et al. Dynamics of the $4 \mathrm{D}$ genome during in vivo lineage specification and differentiation. Nat. Commun. 11, 2722 (2020).

19. Shin, H. Y. et al. Hierarchy within the mammary STAT5-driven Wap superenhancer. Nat. Genet. 48, 904-911 (2016).

20. Li, P. et al. STAT5-mediated chromatin interactions in superenhancers activate IL-2 highly inducible genes: Functional dissection of the Il2ra gene locus. Proc. Natl Acad. Sci. USA 114, 12111-12119 (2017).

21. Metser, G. et al. An autoregulatory enhancer controls mammary-specific STAT5 functions. Nucleic Acids Res. 44, 1052-1063 (2016).

22. Zeng, X., Willi, M., Shin, H. Y., Hennighausen, L. \& Wang, C. Lineage-specific and non-specific cytokine-sensing genes respond differentially to the master regulator STAT5. Cell Rep. 17, 3333-3346 (2016).

23. Liu, X., Robinson, G. W., Gouilleux, F., Groner, B. \& Hennighausen, L. Cloning and expression of Stat5 and an additional homologue (Stat5b) involved in prolactin signal transduction in mouse mammary tissue. Proc. Natl Acad. Sci. USA 92, 8831-8835 (1995).

24. Zhang, Y., Laz, E. V. \& Waxman, D. J. Dynamic, sex-differential STAT5 and BCL6 binding to sex-biased, growth hormone-regulated genes in adult mouse liver. Mol. Cell Biol. 32, 880-896 (2012).

25. Khaled, W. T. et al. The IL-4/IL-13/Stat6 signalling pathway promotes luminal mammary epithelial cell development. Development 134, 2739-2750 (2007).
26. Robinson, G. W. et al. Coregulation of genetic programs by the transcription factors NFIB and STAT5. Mol. Endocrinol. 28, 758-767 (2014).

27. Dinh, D. T. et al. Tissue-specific progesterone receptor-chromatin binding and the regulation of progesterone-dependent gene expression. Sci. Rep. 9, 11966 (2019).

28. Humphreys, R. C., Lydon, J., O’Malley, B. W. \& Rosen, J. M. Mammary gland development is mediated by both stromal and epithelial progesterone receptors. Mol. Endocrinol. 11, 801-811 (1997).

29. Humphreys, R. C., Lydon, J. P., O’Malley, B. W. \& Rosen, J. M. Use of PRKO mice to study the role of progesterone in mammary gland development. $J$. Mammary Gland Biol. Neoplasia 2, 343-354 (1997).

30. Lain, A. R., Creighton, C. J. \& Conneely, O. M. Research resource progesterone receptor targetome underlying mammary gland branching morphogenesis. Mol. Endocrinol. 27, 1743-1761 (2013).

31. Palaniappan, M. et al. The genomic landscape of estrogen receptor a binding sites in mouse mammary gland. PLoS ONE 14, e0220311 (2019).

32. Burdon, T. G., Maitland, K. A., Clark, A. J., Wallace, R. \& Watson, C. J. Regulation of the sheep beta-lactoglobulin gene by lactogenic hormones is mediated by a transcription factor that binds an interferon-gamma activation site-related element. Mol. Endocrinol. 8, 1528-1536 (1994).

33. Kingsley-Kallesen, M. et al. The mineralocorticoid receptor may compensate for the loss of the glucocorticoid receptor at specific stages of mammary gland development. Mol. Endocrinol. 16, 2008-2018 (2002).

34. Reichardt, H. M. et al. Mammary gland development and lactation are controlled by different glucocorticoid receptor activities. Eur. J. Endocrinol. 145, 519-527 (2001).

35. Conneely, O. M., Mulac-Jericevic, B., Lydon, J. P. \& De Mayo, F. J. Reproductive functions of the progesterone receptor isoforms: lessons from knock-out mice. Mol. Cell Endocrinol. 179, 97-103 (2001).

36. Buser, A. C. et al. Progesterone receptor directly inhibits $\beta$-casein gene transcription in mammary epithelial cells through promoting promoter and enhancer repressive chromatin modifications. Mol. Endocrinol. 25, 955-968 (2011).

37. Mukhopadhyay, S. S., Wyszomierski, S. L., Gronostajski, R. M. \& Rosen, J. M. Differential interactions of specific nuclear factor I isoforms with the glucocorticoid receptor and STAT5 in the cooperative regulation of WAP gene transcription. Mol. Cell Biol. 21, 6859-6869 (2001).

38. Lee, H. K. et al. Functional assessment of CTCF sites at cytokine-sensing mammary enhancers using CRISPR/Cas9 gene editing in mice. Nucleic Acids Res. 45, 4606-4618 (2017).

39. Gaffney, D. J. Mapping and predicting gene-enhancer interactions. Nat. Genet 51, 1662-1663 (2019).

40. Hirabayashi, S. et al. NET-CAGE characterizes the dynamics and topology of human transcribed cis-regulatory elements. Nat. Genet. 51, 1369-1379 (2019).

41. Fulco, C. P. et al. Activity-by-contact model of enhancer-promoter regulation from thousands of CRISPR perturbations. Nat. Genet. 51, 1664-1669 (2019)

42. Chen, H. et al. Dynamic interplay between enhancer-promoter topology and gene activity. Nat. Genet. 50, 1296-1303 (2018)

43. Jung, I. et al. A compendium of promoter-centered long-range chromatin interactions in the human genome. Nat. Genet. 51, 1442-1449 (2019).

44. Bolger, A. M., Lohse, M. \& Usadel, B. Trimmomatic: a flexible trimmer for Illumina sequence data. Bioinformatics 30, 2114-2120 (2014).

45. Langmead, B., Trapnell, C., Pop, M. \& Salzberg, S. L. Ultrafast and memory efficient alignment of short DNA sequences to the human genome. Genome Biol. 10, R25 (2009).

46. Masella, A. P. et al. BAMQL: a query language for extracting reads from BAM files. BMC Bioinforma. 17, 305 (2016).

47. Heinz, S. et al. Simple combinations of lineage-determining transcription factors prime cis-regulatory elements required for macrophage and B cell identities. Mol. Cell 38, 576-589 (2010).

48. Ramirez, F. et al. deepTools2: a next generation web server for deepsequencing data analysis. Nucleic Acids Res. 44, W160-W165 (2016).

49. Thorvaldsdottir, H., Robinson, J. T. \& Mesirov, J. P. Integrative Genomics Viewer (IGV): high-performance genomics data visualization and exploration. Brief. Bioinform. 14, 178-192 (2013).

50. Zhang, Y. et al. Model-based analysis of ChIP-Seq (MACS). Genome Biol. 9, R137 (2008)

51. Quinlan, A. R. \& Hall, I. M. BEDTools: a flexible suite of utilities for comparing genomic features. Bioinformatics 26, 841-842 (2010).

52. Love, M. I., Huber, W. \& Anders, S. Moderated estimation of fold change and dispersion for RNA-seq data with DESeq2. Genome Biol. 15, 550 (2014).

53. Dobin, A. et al. STAR: ultrafast universal RNA-seq aligner. Bioinformatics 29, 15-21 (2013)

54. Anders, S., Pyl, P. T. \& Huber, W. HTSeq-a Python framework to work with high-throughput sequencing data. Bioinformatics 31, 166-169 (2015).

55. Huber, W. et al. Orchestrating high-throughput genomic analysis with Bioconductor. Nat. Methods 12, 115-121 (2015). 
56. Risso, D., Ngai, J., Speed, T. P. \& Dudoit, S. Normalization of RNA-seq data using factor analysis of control genes or samples. Nat. Biotechnol. 32, 896-902 (2014).

57. Wickham, H. Ggplot2: elegant graphics for data analysis, viii, p. 212 (Springer, New York, 2009).

58. Willi, M. et al. Facultative CTCF sites moderate mammary super-enhancer activity and regulate juxtaposed gene in non-mammary cells. Nat. Commun. 8 , 16069 (2017).

59. Boeva, V. Analysis of genomic sequence motifs for deciphering transcription factor binding and transcriptional regulation in eukaryotic cells. Front Genet. 7, 24 (2016).

60. Zeng, X. et al. The interdependence of mammary-specific super-enhancers and their native promoters facilitates gene activation during pregnancy. Exp. Mol. Med 52, 682-690 (2020).

\section{Acknowledgements}

We thank Ilhan Akan, Sijung Yun and Harold Smith from the NIDDK genomics core for NGS. This work utilized the computational resources of the NIH HPC Biowulf cluster (http://hpc.nih.gov). This work was supported by the Intramural Research Programs (IRPs) of National Institute of Diabetes and Digestive and Kidney Diseases (NIDDK) and National Heart, Lung, and Blood Institute (NHLBI).

\section{Author contributions}

H.K.L. and L.H. designed the study. C.L. generated mutant mice. H.K.L. and T.K. established mutant mouse lines. H.K.L. performed experiments and data analysis. H.K.L. and M.W. performed computational analysis. H.K.L. and L.H. supervised the study and wrote the manuscript. All authors approved the final version.

\section{Competing interests}

The authors declare no competing interests.

\section{Additional information}

Supplementary information The online version contains supplementary material available at https://doi.org/10.1038/s41467-021-22500-w.

Correspondence and requests for materials should be addressed to H.K.L. or L.H.

Peer review information Nature Communications thanks Monique Rijnkels, Christine Watson and the other, anonymous, reviewer(s) for their contribution to the peer review of this work. Peer reviewer reports are available.

Reprints and permission information is available at http://www.nature.com/reprints

Publisher's note Springer Nature remains neutral with regard to jurisdictional claims in published maps and institutional affiliations.

\begin{abstract}
(c) (i)
Open Access This article is licensed under a Creative Commons Attribution 4.0 International License, which permits use, sharing, adaptation, distribution and reproduction in any medium or format, as long as you give appropriate credit to the original author(s) and the source, provide a link to the Creative Commons license, and indicate if changes were made. The images or other third party material in this article are included in the article's Creative Commons license, unless indicated otherwise in a credit line to the material. If material is not included in the article's Creative Commons license and your intended use is not permitted by statutory regulation or exceeds the permitted use, you will need to obtain permission directly from the copyright holder. To view a copy of this license, visit http://creativecommons.org/ licenses/by/4.0/.
\end{abstract}

This is a U.S. Government work and not under copyright protection in the US; foreign copyright protection may apply 2021 